\title{
Laser Nano-Structuring of Pre-Structured Substrates
}

\author{
E.V.Barmina ${ }^{1 *}$, E. Skoulas ${ }^{2}$, E. Stratakis ${ }^{2}$, and G.A. Shafeev ${ }^{1,3}$ \\ ${ }^{1}$ Wave Research Center of A.M. Prokhorov General Physics Institute of the Russian Academy of Sciences, 38, Vavilov \\ street, 119991, Moscow Russian Federation \\ E-mail: barminaev@gmail.com \\ ${ }^{2}$ Institute of Electronic Structure and Laser, Foundation for Research \& Technology—Hellas, (IESL-FORTH), P.O. Box \\ 1527, Heraklion 711 10, Greece \\ ${ }^{3}$ National Research Nuclear University MEPhI (Moscow Engineering Physics Institute), 31, Kashirskoye highway, 115409, \\ Moscow Russian Federation
}

Laser nano-structuring of pre-structured Ni substrate using short and ultra-short laser pulses is experimentally studied. Prestructured samples were prepared using electron beam lithography with final periodic relief with period $300-500 \mathrm{~nm}$ and sinusoidal profile. The morphology of laser-produced nanostructures strongly depends on the respective orientation of prestructured grating and polarization vector of the laser beam. Surface Electromagnetic Waves induced in Ni substrate that interfere with laser beam are strongly dumped if the polarization vector of laser light is perpendicular to the initial e-beam grating. The morphology of laser-produced nanostructures also depends on the laser fluence of the substrate. Laser nano-

structuring of Ni using a phase plate in the laser beam results in very specific morphology of the irradiated surface.

DOI: 10.2961/jlmn.2018.01.0002

Keywords: laser ablation, LIPSS, nanostructures, surface electromagnetic waves

\section{Introduction}

Interaction of laser radiation with solid surfaces is always accompanied by the change of its morphology. The first structures that were observed since the invention of lasers are so called Laser-Induced Periodic Surface Structures (LIPSS) or ripples [1-3]. These periodic structures are due to the interference of the linearly polarized laser beam and Surface Electromagnetic Waves (SEW) induced in the surface of the target by laser beam. The amplitude of SEW decreases exponentially from both side of the surface (in air and in metal). These waves are always in the same phase with laser light, and the resulting intensity distribution is close to sinusoidal. If the intensity of radiation is sufficiently high in the maxima of the interference pattern to alter physical properties of the surface (melting, evaporation, etc.) then the pattern is "recorded" on the surface of the target. The period of LIPSS is determined by the laser wavelength $\lambda$, angle of incidence $\phi$, and refractive index of the medium surrounding the target $n: d=\lambda /(n \pm \sin \phi)$. For small $\lambda$ values the period of ripples lies in nanometer range [1].

Ripples should be distinguished from another type of nanostructures, namely, so called self-organized nanostructures. They are formed under exposure of the surface of metals by laser pulses with duration less than 1 ns. Their period is determined by the melt thickness and surface tension of the melt. These nanostructures look like isolated hillocks with average distance between them of order $100 \mathrm{~nm}$. Their morphology depends neither on the polarization of laser radiation nor on laser wavelength [4 6]. In some cases, however, self-organized nanostructures may co-exist with ripples. Self-organized nanostructures are situated on the tops of ripples.

LIPSS are sensitive to the state of the surface. Dumping of SEW is strongly affected by defects of the surface, such as scratches, pits, etc. The presence of defects, in turn, affects the morphology of LIPSS. Also, existence and propagation of SEW requires the conductive surface. There is certain discrepancy between this requirement and formation of ripples on dielectrics, such as $\mathrm{BaF}_{2}$ [7] and $\mathrm{SiC}[8,9]$. Formation of ripples in these cases may be explained by two-photon absorption of laser radiation. Indeed, in case of two-photon absorption the conductivity band of dielectrics can be populated by electrons, and the surface becomes conductive at least during the laser pulse or shortly after it. That is why the ripples period in case of $\mathrm{SiC}$ is twice less than $\lambda$ and is approximately $\lambda / 2$.

It is of certain interest to elucidate the influence of initial pre-structure on the morphology of ripples produced by laser ablation with either linearly polarized laser radiation or with polarization that varies inside the laser beam. The latter can be achieved using special phase plates. The aim of this work is to determine the morphology of surface nanostructures with initial regular pre-structuring with period close to that of ripples on smooth surface.

\section{Experimental}

Nickel foil of $100 \mu \mathrm{m}$ thickness was used as a target. The foil was fixed on a computer-driven X-Y stage. The target was exposed in air to radiation of a $\mathrm{Yb}: \mathrm{KGW}$ laser at wavelength of $1030 \mathrm{~nm}$ with pulse duration of $160 \mathrm{fs}$ at repetition rate of $1 \mathrm{kHz}$. The morphology of $\mathrm{Ni}$ target was characterized by Scanning Electron Microscope with field emission. Some area of the Ni target was pre-structured using electron beam lithography. Typical view of prestructured surface is presented in Fig. 1. The period of sinusoidal grooves is about $1300 \mathrm{~nm}$, as it follows from AFM view. 


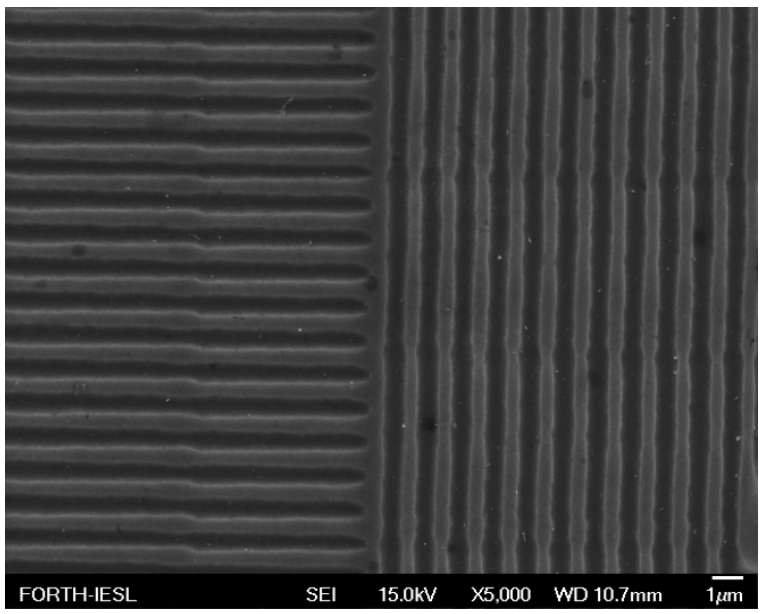

a

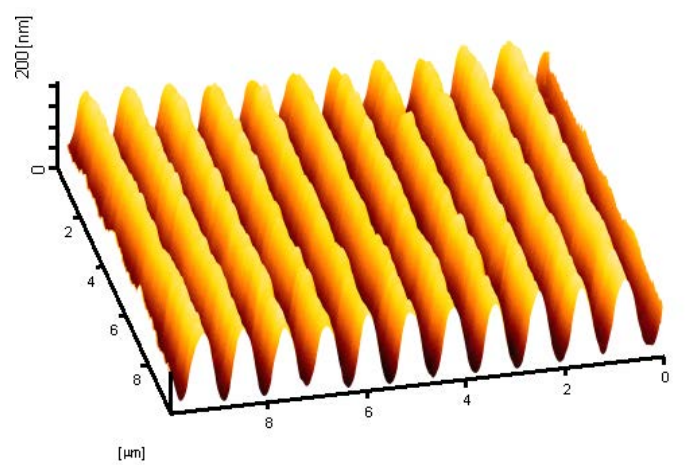

b

Fig. 1. Pre-structured Ni surface. One can see two areas with different orientation of initial relief. Scale bar denotes $1 \mu \mathrm{m}$ (a). Atomic Force Microscope view of the pre-structured surface (b). The amplitude of sinusoidal grooves is around $200 \mathrm{~nm}$.

Initially linearly polarized laser beam was transformed into azimuthal polarization with the help of so called Kazansky plate [10]. Direction of polarization after the plate and the intensity distribution are presented in Fig. 2.
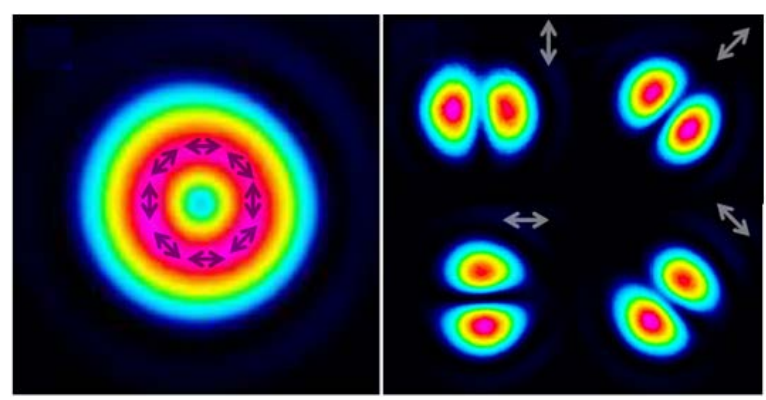

Fig. 2. Polarization distribution (left) and intensity distribution of the laser beam (right) after Kazansky plate. In the work the intensity distribution shown in the upper right corner was used. Illustration taken from [10].

\section{Results and discussion}

Fig. 3 shows the surface relief formed after laser ablation of the Ni target on differently pre-structures surfaces. LIPSS are oriented in the direction perpendicular to the polarization vector of the laser beam (yellow arrow).

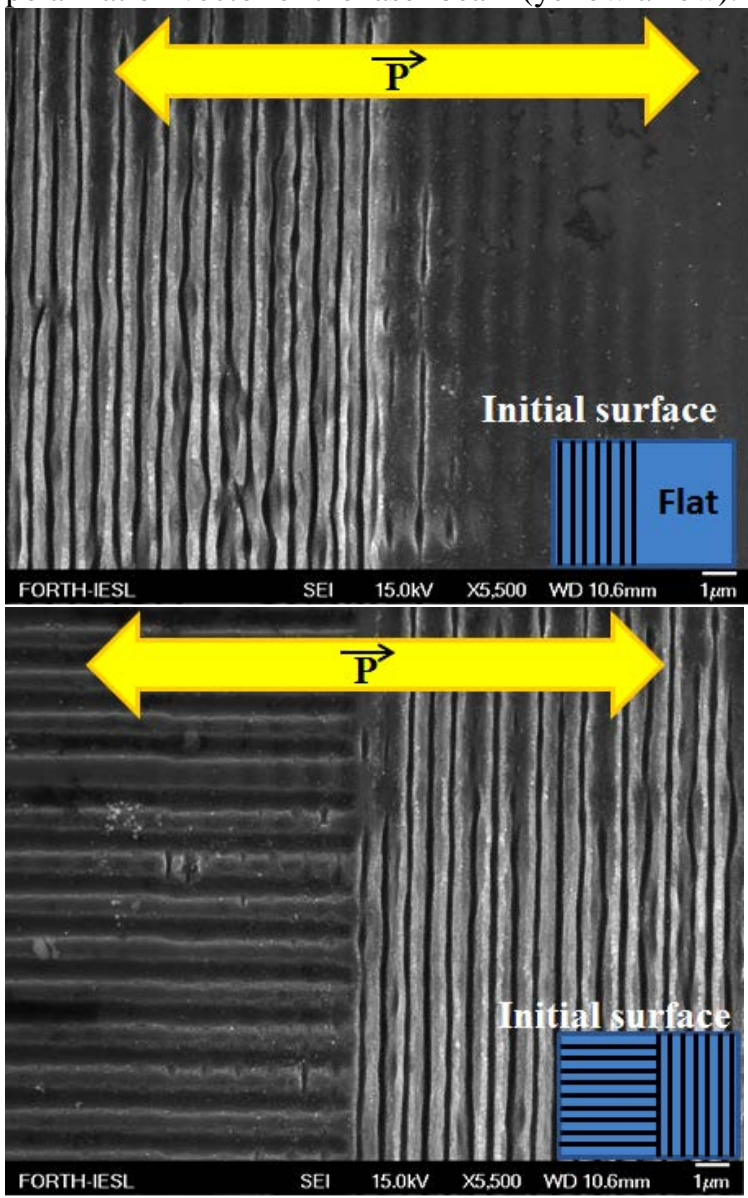

Fig. 3. Relief formed after ablation of flat and pre-structured surface (a). Relief formed after ablation of the surface prestructured in two perpendicular directions (b). Yellow arrow indicates the polarization vector of laser beam. Scale bar denotes $1 \mu \mathrm{m}$. Laser fluence of $0.1 \mathrm{~J} / \mathrm{cm}^{2}$.

One can see that on the initial flat surface there are only traces of ripples left by laser beam. On the contrary, the effect of laser beam is clearly seen on the pre-structured surface when the direction of initial relief coincides with that of SEW (Fig. 3, a). Deep grooves are formed on tops of the initial relief. In other words, this relief of prestructured surface is suitable for SEW propagation. Similarly, the same direction is favorable for SEW propagation (Fig. 3,b, right part). However, at perpendicular orientation of pre-structured grooves almost no traces of the laser beam are seen on the surface (left part of Fig. 3, b). This type of pre-structuring apparently provides high surface dumping for SEW.

Closer view in Fig. 3, a shows that the period of the relief formed on the favorably pre-structured surface 
$(650 \mathrm{~nm})$ is two times less than the laser wavelength of $1030 \mathrm{~nm}$ and almost than 2 times less than the period of initial pre-structure of $1260 \mathrm{~nm}$. This can be interpreted as the generation of the second spatial harmonics of surface relief. This is probably due to the proximity of the period of initial sinusoidal structure to the period of LIPSS.

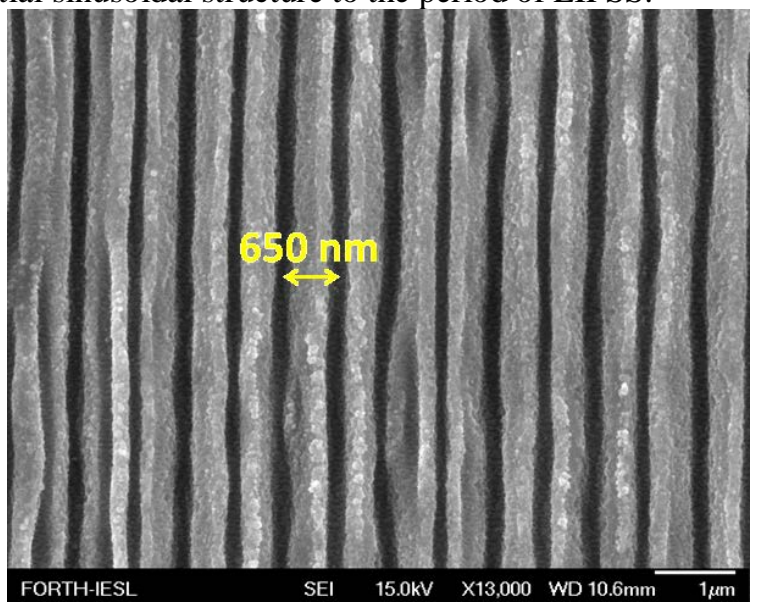

Fig. 4. Enlarged view of the surface with "favorable" prestructuring.. Scale bar denotes $1 \mu \mathrm{m}$.

Dumping of the SEW on "unsuitable" orientation of the initial structure (Fig. 3, b) can be partially compensated by the increased laser fluence. This is demonstrated in Fig. 5.

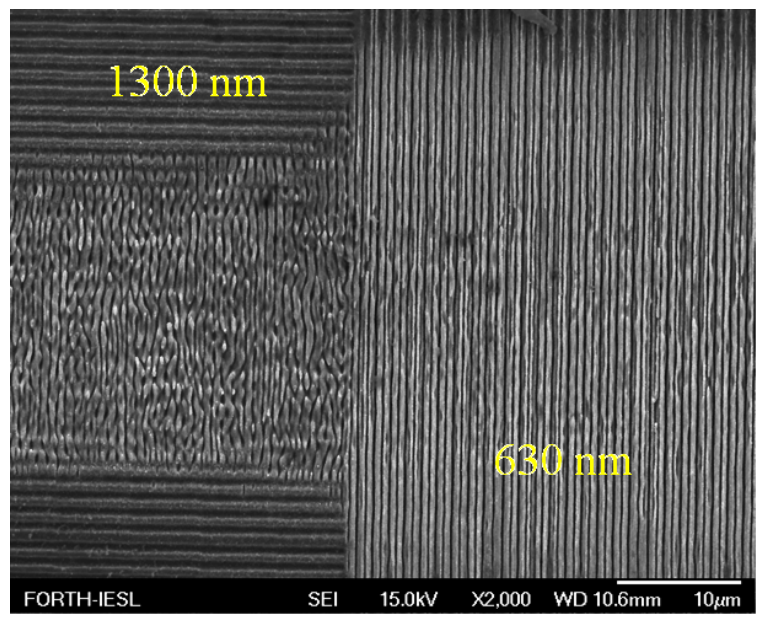

Fig. 5. Relief formed after ablation of the surface pre-structured in two perpendicular directions at increased laser fluence of 0.5 $\mathrm{J} / \mathrm{cm}^{2}$. Scale bar denotes $10 \mu \mathrm{m} .1300 \mathrm{~nm}$ is the period of prestructured pattern, $630 \mathrm{~nm}$ is the period of LIPSS produced on the pre-structured target.

The resulting relief is slightly irregular but is wellpronounced and follows the direction of ripples that would be produced on a flat surface of $\mathrm{Ni}$. It is formed mostly in the central part of the Gaussian laser beam. Therefore, dumping of SEW can be compensated by higher laser fluence.

If the polarization of the laser beam is not perpendicular or parallel to the pre-structured relief then the resulting structures takes on the orientation of ripples perpendicular to the polarization independently on the prestructured surface. This is illustrated in Fig. 6.

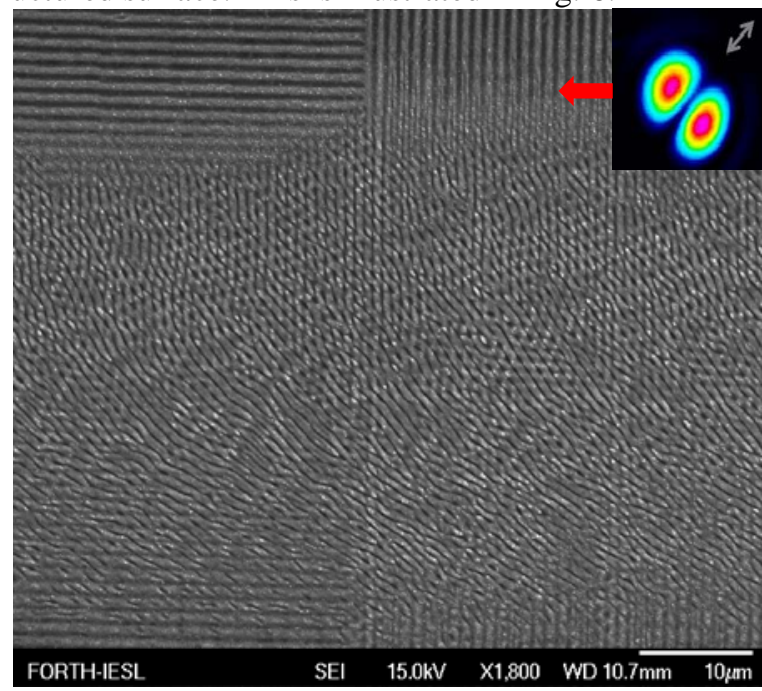

Fig. 6. Interaction of laser beam after passing Kazansky plate with pre-structured surface. The inset shows the intensity distribution and polarization direction of the laser beam. The scan of the laser beam was performed from right to left as shown by red arrow. Scale bar denotes $10 \mu \mathrm{m}$, laser fluence of $0.5 \mathrm{~J} / \mathrm{cm}^{2}$.

One can see that LIPSS "ignore" the pre-structured relief, the whole direction of the structures are as usually perpendicular to the polarization vector of the laser beam. However, the pre-structured surface shown at the right of Fig. 6 intersects with the incident laser beam in a specific way producing rhomboidal nanostructures. Their morphology is shown in Fig. 7.

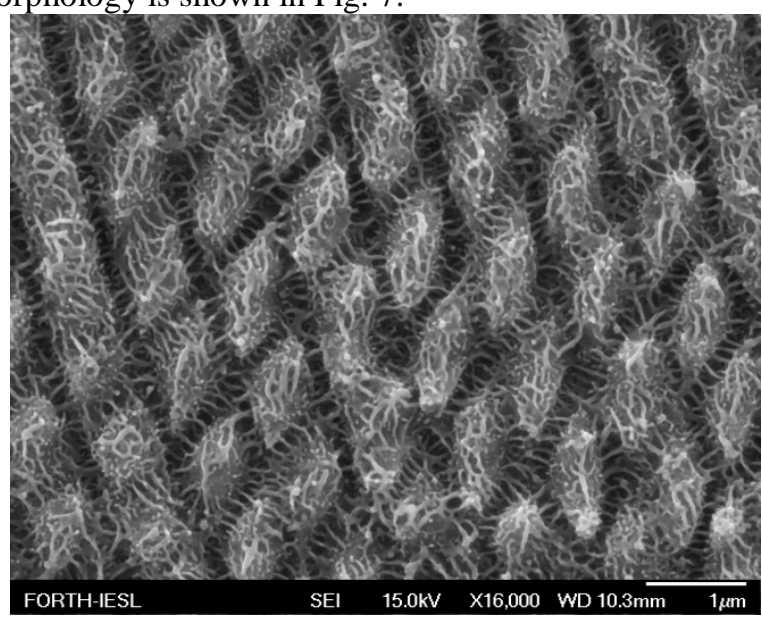

Fig. 7. Intersection of pre-patterned structure with the laser beam polarized at $45^{\circ}$ with respect to initial grooves (Kazansky plate).

One can see the ultra-fine structures in between the rhombs of the relief. In fact, such ultra-fine structures formation has been reported in many previous papers (see, for example [7, 8, 11]). They are often observed inside the ripples and are always perpendicular to the direction of ripples. These ultra-fine structures could not be assigned to SEW since their period is of order of $100 \mathrm{~nm}$, which is by far smaller than the laser wavelength. Presumably, these ultra-fine structures are due to the development of thermo- 
capillary instability of the melt bath. The melt bath thickness under ablation with short and ultra-short laser pulses is of order of several tens of nanometers. The formation of ultra-fine structures is due to high temperature gradients along the melt and to the depth of the melt. The coefficient of surface tension decreases with temperature. This dependence is the driving force of surface perturbation. The theoretical model of the process of ultra-fine structures formation is now under preparation.

\section{Conclusion}

Thus, the formation of ripples on periodically prestructured Ni targets has been experimentally studied. It is found for the first time that there are two different orientations of pre-structuring that affect differently the resulting nano-relief. Orientation with grooves perpendicular to polarization vector of the laser beam leads to formation of LIPSS with period $\lambda / 2$, where $\lambda$ is laser wavelength. In this sense one may speak about generation of the second spatial harmonics of LIPSS. Orientation with grooves parallel to polarization vector of the laser beam dumps the SEW and no LIPSS are observed at low laser fluence of $0.1 \mathrm{~J} / \mathrm{cm}^{2}$. At higher fluence of $0.5 \mathrm{~J} / \mathrm{cm}^{2}$ LIPSS appear but their period is about $\lambda=1030 \mathrm{~nm}$. Phase S-plate with polarization vector oriented at 45 with respect to initial pre-structuring produces LIPSS whose orientation is independent on the orientation of initial grooves. Its period is about $\lambda$. One may conclude that initial pre-structuring drastically alters resulting LIPSS morphology.

\section{Acknowledgements}

The work was partially supported by Russian Foundation of Basic Researches, grants 15-02-04510_a, 16-0201054_a RF President grant MK-3606.2017.2.

\section{References}

[1] M. J. Birnbaum: J. Appl. Phys., 36, (1965) 3688.

[2] S.A. Akhmanov, V.I. Emel'yanov, N.I. Koroteev, V.N. Seminogov: J. Usp. Fiz. Nauk., 147, (1985) 675

[3] J. F. Young, J. S. Preston, H. M. Van Driel, J. E. Sipe: Phys. Rev. B, 27, (1983) 1141.

[4] E. V. Zavedeev, A.V. Petrovskaya, A.V. Simakin, G. A. Shafeev: Quant. Electr., 36, (2006) 978.

[5] S. Lau Truong, G. Levi, F. Bozon-Verduraz, A. V. Petrovskaya, A. V. Simakin, G. A. Shafeev: J. Appl. Phys. A, 89, (2007) 373

[6] E. Stratakis, V. Zorba, M. Barberoglou, C. Fotakis, G.A. Shafeev: Appl. Surf. Sci., 255, (2009) 5346.

[7] F. Costache, M. Henyk, J. Reif: Appl. Surf. Sci., 208209, (2003) 486.

[8] E.V. Barmina, A.A. Serkov, G.A. Shafeev: Quant. Electr., 43, (2013) 1091.

[9] E. V. Barmina, A. A. Serkov, G. A Shafeev, E. Stratakis, C. Fotakis: Phys. Wave Phenom., 22, (2014) 15.

[10] M. Beresna, M. Gecevičius, P. G. Kazansky: Opt. Mat. Express., 1, (2011) 783.

[11] X. Sedao, M. V. Shugaev, C. Wu, T. Douillard, C. Esnouf, C. Maurice, S. Reynaud, F. Pigeon, Florence G., L. 